\title{
SEPARATION OF ROOTS AND OSCILLATION IN ORDINARY LINEAR DIFFERENTIAL EQUATIONS OF SECOND ORDER
}

\author{
SHLOMO BREUER AND DAVID GOTTLIEB
}

\begin{abstract}
New criteria for the oscillatory or nonoscillatory behavior of ordinary, linear differential equations of second order and the location of their roots are obtained with the aid of new types of Sturmian majorant comparison equations. Several known results are obtained as special cases under less stringent conditions.
\end{abstract}

1. Introduction. The oscillatory properties of ordinary differential equations of the second order and the approximate location of their zeros have received widespread attention in the literature. Recently, Swanson [1] has compiled many of the known results in book form. The principal method of investigation is the use of the classical form or some modern variant of the Sturm Separation Theorem. Thus one must find an equation which majorizes the given one in the Sturmian sense in order to be able to compare the two and obtain the desired information. A typical comparison equation is the Euler equation whose use, of course, is limited due to its very special nature.

In this paper we introduce two new types of comparison equations for second order linear differential equations-which are indeed generalizations of the Euler equation-whose solutions are known and which can be associated for comparison purposes with the equation under investigation. These associated comparison equations have been previously discussed by the authors in connection with the transformation of ordinary differential equations into equations with constant coefficients [2]. With the aid of these new comparison equations we are able to find new criteria for oscillation and nonoscillation as well as approximate the location of zeros inside finite and infinite intervals.

Moreover, several known results which have been obtained by a fairly complicated analysis are recovered here as special cases and under less stringent conditions. 1970.

Rereived by the editors October 12, 1970 and, in revised form, November 23,

AMS 1969 subject classifications. Primary 3420; Secondary 3431.

Key words and phrases. Differential equations of second order, Sturm Separation Theorem, separation of roots, oscillations, location of roots, comparison equations.

Copyright (C) 1971, American Mathematical Society 
2. Separation of roots and oscillation theorems. We begin with two lemmas which form the basis for all subsequent results.

Lемма 1. Let the differential equation

$$
\left[a(x) u^{\prime}\right]^{\prime}+\frac{k_{1}}{a(x)[p(x)]^{2}} u=0, \quad x \in(\alpha, \beta),
$$

hold in some open interval $(\alpha, \beta)$, where $a(x)$ is a positive, continuously differentiable function and $k_{1}$ a positive constant. Then the general solution of (1) is given by

$$
u(x)=A_{1}[p(x)]^{m_{1}}+A_{2}[p(x)]^{m_{2}},
$$

where

$$
m_{1,2}=\frac{1}{2} \pm\left(\frac{1}{4}-k_{1}\right)^{1 / 2}, \quad p(x)=k_{2}+\int_{\alpha}^{x} \frac{d t}{a(t)},
$$

$A_{1}$ and $A_{2}$ being arbitrary constants and $k_{2}$ a nonnegative constant.

LEMma 2. Let the differential equation

$$
\left\{\frac{K_{1}[q(x)]^{2}}{c(x)} z^{\prime}\right\}^{\prime}+c(x) z=0, \quad x \in(\alpha, \beta),
$$

hold in some open interval $(\alpha, \beta)$, where $c(x)$ is a positive, continuously differentiable function and $K_{1}$ a positive constant. Then the general solution of (4) is given by

$$
z(x)=B_{1}[q(x)]^{n_{1}}+B_{2}[q(x)]^{n_{2}}
$$

where

(6) $\quad n_{1,2}=-1 / 2 \pm\left[1 / 4-\left(1 / K_{1}\right)\right]^{1 / 2}, \quad q(x)=K_{2}+\int_{\alpha}^{x} c(t) d t$,

$B_{1}$ and $B_{2}$ being arbitrary constants and $K_{2}$ a nonnegative constant.

Lemmas 1 and 2 follow directly from Corollary 4.1 case (i) in [2]. They can of course be verified directly, by substitution.

REMARK 1. If $k_{1}>\frac{1}{4}$, then $m_{1}$ and $m_{2}$ are complex and are given by

$$
m_{1,2}=1 / 2 \pm \gamma i, \quad \gamma=\left(k_{1}-1 / 4\right)^{1 / 2} .
$$

In that case, (2) gives way to

$$
u(x)=[p(x)]^{1 / 2}\left\{A_{1} \sin [\gamma \log p(x)]+A_{2} \cos [\gamma \log p(x)]\right\} .
$$


Similarly, if $K_{1}<4$, then $n_{1}$ and $n_{2}$ are complex and are given by

$$
n_{1,2}=-\frac{1}{2} \pm \delta i, \quad \delta=\left(1 / K_{1}-\frac{1}{4}\right)^{1 / 2},
$$

so that (5) takes the form

$$
z(x)=[q(x)]^{-1 / 2}\left\{B_{1} \sin [\delta \log q(x)]+B_{2} \cos [\delta \log q(x)]\right\} .
$$

REMARK 2. If we let $k_{1}$ and $k_{2}$ tend to infinity in such a way that $\left(k_{2}^{2} / k_{1}\right)$ tends to a positive constant, $d^{-2}$, then the general solution of (1) appears as

$$
u(x)=A_{1} \sin \left[d \int_{\alpha}^{x} \frac{d t}{a(t)}\right]+A_{2} \cos \left[d \int_{\alpha}^{x} \frac{d t}{a(t)}\right] .
$$

Similarly, if analogous conditions are met by $\left(1 / K_{1}\right), K_{2}$ and some positive constant, $D^{-2}$, respectively, then the general solution of (4) appears as

$$
z(x)=B_{1} \sin \left[D \int_{\alpha}^{x} c(t) d t\right]+B_{2} \cos \left[D \int_{\alpha}^{x} c(t) d t\right] .
$$

It is readily seen that Remark 2 is a direct consequence of case (iii) of Corollary 4.1 in [2].

Next we define, for future reference, the following four quantities.

$$
\begin{aligned}
v_{*} & =\inf _{x \in(\alpha, \beta)} a(x) c(x)[p(x)]^{2}, \\
v^{*} & =\sup _{x \in(\alpha, \beta)} a(x) c(x)[p(x)]^{2}, \\
V_{*} & =\inf _{x \in(\alpha, \beta)} a(x) c(x)[q(x)]^{-2}, \\
V^{*} & =\sup _{x \in(\alpha, \beta)} a(x) c(x)[q(x)]^{-2} .
\end{aligned}
$$

We are now ready for the following theorem.

THEOREM 1. Let the differential equation

$$
\left[a(x) y^{\prime}\right]^{\prime}+c(x) y=0, \quad x \in(\alpha, \beta),
$$

hold in some open interval $(\alpha, \beta)$, where $c(x)$ is continuous (not necessarily positive), and $a(x)$ is as in Lemma 1 . Then the following results hold.

(a) If $v^{*}<1 / 4$, for some $k_{2} \geqq 0$, then $y(x)$ vanishes at most once in $(\alpha, \beta)$.

(b) If $v_{*}>1 / 4$, for some $k_{2} \geqq 0$, then $y(x)$ vanishes between any two 
consecutive zeros of $u(x)$ as given by (8), with $k_{1}=v_{*}$. Since we can easily find $k_{2}, A_{1}$, and $A_{2}$ such that $u(\alpha)=u(\beta)=0$, it is clear that $y(x)$ vanishes at some point.

Proof. In (1), choose $k_{1}=v^{*}$ and compare (1) with (14). By the classical Sturm Comparison Theorem, the solution $u(x)$ of (1), given by (2) for $k_{1}<1 / 4$, must vanish between any two consecutive zeros of $y(x)$. But for $k_{1}<1 / 4, u(x)$ vanishes at most once in $(\alpha, \beta)$ and hence so does $y(x)$. This proves (a).

To prove (b), choose $k_{1}=v_{*}$ in (1). The Sturm Comparison Theorem readily shows that $y(x)$ vanishes between any two consecutive zeros of $u(x)$, which for $k_{1}>1 / 4$ is given by (8). This completes the proof of Theorem 1 .

The next theorem, based on (4), is analogous to Theorem 1.

THEOREM 2. Let (14) hold in $(\alpha, \beta)$ with both $a(x)$ and $c(x)$ continuously differentiable, positive functions. Then the following results hold.

(a) If $V^{*}<4$, for some $K_{2} \geqq 0$, then $y(x)$ vanishes between any two consecutive zeros of $z(x)$ as given by (10), with $K_{1}=V^{*}$. As in Theorem $1(\mathrm{~b})$, it is clear that $y(x)$ vanishes at some point.

(b) If $V_{*}>4$, for some $K_{2} \geqq 0$, then $y(x)$ vanishes at most once in $(\alpha, \beta)$.

Proof. In (4), choose $K_{1}=V^{*}$ for case (a) and $K_{1}=V_{*}$ for case (b), and proceed exactly as in Theorem 1.

We shall see next that Theorems 1 and 2 contain some known results as special cases. Moreover, the method of proof employed here enables us to extend some known results as well. We shall be concerned with semi-infinite intervals for which $\beta=\infty$.

In the following corollaries we shall use the symbols $w_{*}, w^{*}, W_{*}$ and $W^{*}$ to denote the values of the right-hand sides of (13), respectively, after sup and inf have been replaced with lim sup and lim inf.

Corollary 1. Let $a(x)$ and $c(x)$ be as in Theorem 1 .

(a) If $\int_{\alpha}^{\infty}(d t / a(t))=\infty$ and $w_{*}>1 / 4$, then

(i) The solution $y(x)$ of (14) is oscillatory.

(ii) The zeros of $y(x)$ occur, asymptotically as $x \rightarrow \infty$, between any two consecutive zeros of (8), in which $k_{1}=w_{*}$, and we may take $k_{2}=0$.

(b) If $w^{*}<1 / 4, y(x)$ is nonoscillatory.

For $a(x) \equiv 1$, case (a) (i) and case (b) reduce to the Hille-Kneser Theorem [3], [1, Theorem 2.41]. Case (a)(ii) appears to be a new result. 
COROLlary 2. Let $a(x)$ and $c(x)$ be as in Theorem 2.

(a) If $\int_{\alpha}^{\infty} c(t) d t=\infty$ and $W^{*}<4$, then

(i) The solution $y(x)$ of $(14)$ is oscillatory.

(ii) The zeros of $y(x)$ occur, asymptotically as $x \rightarrow \infty$, between any two consecutive zeros of (10), in which $K_{1}=W^{*}$, and we may take $K_{2}=0$.

(b) If $W_{*}>4, y(x)$ is nonoscillatory.

REMARK 3. If in Corollaries 1 and 2 the conditions $w_{*}>1 / 4$ and $W^{*}<4$ can be replaced with $v_{*}>1 / 4$ and $V^{*}<4$, respectively, the conclusions about the interlacing of the zeros of $y(x)$ will clearly hold throughout the interval $(\alpha, \infty)$.

CoROllaRY 3. Let $a(x)$ and $c(x)$ be continuously differentiable functions in $(\alpha, \infty)$, let $a>0$, and c nonnegative. Let

$$
\lim _{x \rightarrow \infty} a\left[(a c)^{-1 / 2}\right]^{\prime}=L .
$$

(a) If $\int_{\alpha}^{\infty}(d t / a(t))=\infty$ and $0 \leqq L<2$, then

(i) The solution $y(x)$ of (14) is oscillatory.

(ii) The zeros of $y(x)$ occur, asymptotically as $x \rightarrow \infty$, between any two consecutive zeros of (8), in which $k_{1}=L^{-2}$ provided $L \neq 0$, and we may take $k_{2}=0$.

(b) If $L>2, y(x)$ is nonoscillatory.

Proof. Let $L<2$. From (15) we infer the existence of $x_{0}$ such that for every $\epsilon>0$ and $x>x_{0}(\epsilon)$,

$$
a\left[(a c)^{-1 / 2}\right]^{\prime}<L+\epsilon<2,
$$

which implies

$$
(a c)^{-1}<\left\{(L+\epsilon) \int_{\alpha}^{x} \frac{d t}{a(t)}+\left.(a c)^{-1 / 2}\right|_{x=x_{0}}\right\}^{2} .
$$

Consequently,

$$
w_{*}=\lim \inf a c\left[\int_{\alpha}^{x} \frac{d t}{a(t)}\right]^{2}>\frac{1}{(L+\epsilon)^{2}}>\frac{1}{4},
$$

and the result of (a) follows from Corollary 1. The proof of (b) is analogous.

The case $L=0$ has been treated by Potter [4], [1, Theorem 2.57]. Case (a)(i) and case (b) have been obtained by Potter [4], [1, Theorem 2.36], but only with the additional condition, for case (b), that $\int_{\alpha}^{\infty}(d t / a(t))=\infty$, which is not required here. Case (a)(ii) appears to be a new result.

Exactly as we proved Corollary 3, we may also prove the following. 
Corollary 4. Let a $(x)$ and $c(x)$ be as in Corollary 3 , and let

$$
\lim _{x \rightarrow \infty} \frac{1}{c}\left[(a c)^{1 / 2}\right]^{\prime}=M .
$$

(a) If $\int_{\alpha}^{\infty} c(t) d t=\infty$ and $0 \leqq M<2$, then

(i) The solution $y(x)$ of (14) is oscillatory.

(ii) The zeros of $y(x)$ occur, asymptotically as $x \rightarrow \infty$, between any two consecutive zeros of (10), in which $K_{1}=M^{2}$ provided $M \neq 0$, and we may take $K_{2}=0$.

(b) If $M>2$, then $y(x)$ is nonoscillatory.

The next two corollaries are based upon Remark 2, following Lemma 2.

Corollary 5. Let $a(x)$ and $c(x)$ be as in Theorem 1 , and let $a c \leqq H^{2}$ for some positive constant $H^{2}$, in $(\alpha, \infty)$. Then a necessary condition for (14) to be oscillatory is that

$$
\lim _{x \rightarrow \infty} \int_{\alpha}^{x} \frac{d t}{a(t)}=\infty .
$$

If, in addition, $c(x)$ is as in Theorem 2, then a sufficient condition for (14) to be oscillatory is that

$$
\lim _{x \rightarrow \infty} \int_{\alpha}^{x} c(t) d t=\infty .
$$

In that case, the zeros of the solution $y(x)$ of (14) occur, asymptotically as $x \rightarrow \infty$, between any two consecutive zeros of (12), with $D=H^{-1}$.

Proof. Consider the differential equation

$$
\left[a(x) u^{\prime}\right]^{\prime}+\frac{H^{2}}{a(x)} u=0 .
$$

By the Sturm Separation Theorem, and since $a c \leqq H^{2}$, (22) must be oscillatory if (14) is. But by Remark 2, the solution of (22) is given by (11) with $d=H$, for which a necessary condition for oscillation is precisely (20).

To prove the sufficiency condition (21), we consider

$$
\left[\frac{H^{2}}{c(x)} z^{\prime}\right]^{\prime}+c(x) z=0,
$$

whose solution is given by (12) with $D=H^{-1}$. If (21) holds, (12) is 
oscillatory and hence, by the Sturm Separation Theorem, so is (14). The proof of the interlacing of the zeros is now trivial.

We observe that the conditions for oscillation in this corollary have also been obtained by Moore [5], [1, p. 73], but the location of the zeros deduced here appears to be a new result.

Finally, we have the following corollary, analogous to Corollary 5.

CoRollary 6. Let $a(x)$ and $c(x)$ be as in Theorem 2, and let ac $\geqq h^{2}>0$. Then a necessary condition for (14) to be oscillatory is (21) and a sufficient condition for (14) to be oscillatory is (20). In that case, the zeros of (14) occur, asymptotically as $x \rightarrow \infty$, between any two consecutive zeros of (11), with $d=h$.

\section{REFERENCES}

1. C. A. Swanson, Comparison and oscillation theory of linear differential equations, Academic Press, New York and London, 1968.

2. S. Breuer and D. Gottlieb, The reduction of linear ordinary differential equations to equations with constant coefficients, J. Math. Anal. Appl. 32 (1970) 62-76.

3. E. Hille, Non-oscillation theorems, Trans. Amer. Math. Soc. 64 (1948), 234252. MR 10, 376.

4. R. L. Potter, On self adjoint differential equations of second order, Pacific J. Math. 3 (1953), 467-491. MR 15, 32.

5. R. A. Moore, The behaviour of solutions of a linear differential equation of second order, Pacific J. Math. 5 (1955), 125-145. MR 16, 925.

Tel Aviv University, Tel Aviv, Israel 